\title{
Evaluación de factores psicológicos asociados al transporte sustentable*
}

\section{Evaluation of Psychological Factors Associated with Sustainable Transportation}

\author{
Lucía Jaramillo Ramos \\ ORCID: 0000-0002-4231-1515 \\ Johann Streiker Díaz-Marín** \\ ORCID: 0000-0001-5899-4501 \\ Universidad de los Andes, Colombia \\ Recibido: 3 de diciembre de 2019 \\ Revisado: 7 de febrero de 2020 \\ Aceptado: 2 de mayo de 2020
}

\section{Resumen}

Esta investigación surge de la necesidad de encontrar nuevos hábitos de consumo sustentable y está enfocada en la identificación de los factores psicológicos asociados al uso habitual de la bicicleta como un transporte sostenible. Se evaluaron varios grupos de variables en estudiantes universitarios $(N=492)$. Se consultaron los tres predictores de la Teoría de Acción Planeada, algunas características sociodemográficas y los hábitos de transporte. Mediante una taxonomía de modelos lineales se integraron y analizaron estas variables. Los resultados señalan que las personas de mayor edad, mayor actitud y mayor control comportamental percibido son la mejor caracterización significativa que se puede tener de los estudiantes biciusuarios. También se encuentra que el control comportamental percibido es el correlato más fuerte con el uso de la bicicleta. Se discuten las implicaciones para campañas dirigidas a la promoción del uso de la bicicleta en los estudiantes universitarios.

Palabras Clave: Transporte sustentable, psicología ambiental, teoría de acción planeada, hábito.

Artículo de investigación. Citar como: Jaramillo, R. L. y Díaz-Marín, J. S. (2020). Evaluación de factores psicológicos asociados al transporte sustentable. Diversitas: Perspectivas en Psicología, 16(2), 341-352. https://doi.org/10.15332/22563067.6317

Autor de correspondencia: Johann Streiker Díaz-Marín, Universidad de los Andes, Bogotá, Colombia. Correo electrónico: js.diaz16@uniandes. edu.co 


\section{Abstract}

This research arises from the need to find new habits of sustainable consumption, and it is focused on the identification of psychological factors associated with the regular use of the bicycle as a sustainable transportation. Several groups of variables were evaluated in 492 undergraduate students. The three predictors of the Theory of Planned Behavior, some sociodemographic characteristics, and transportation habits were consulted. Through a taxonomy of linear models, these variables were integrated and analyzed. Results indicate that older people, greater attitude, and greater perceived behavioral control are the best significant characterization that can be achieved of bike-user students. We found perceived behavioral control is the strongest correlation with the use of bicycles. Implications for campaigns aimed at promoting the use of bicycles in students are discussed.

Keywords: sustainable transportation, environmental psychology, theory of planned behavior, habit.

\section{Introducción}

Psicólogos y diversos profesionales de las ciencias sociales han realizado un esfuerzo por generar un engranaje entre el conocimiento teórico y su aplicabilidad a problemáticas actuales. Como plantea Baird (2015), gran parte de los retos a los que se enfrenta nuestra sociedad están configurados por componentes en los que las ciencias sociales y del comportamiento pueden aportar, desde la preparación militar hasta el cambio climático. Haciendo referencia a la sostenibilidad, los psicólogos han aportado a la comprensión y promoción de comportamientos proambientales (Gifford, 2007). Como expresa Moser (1998, 2003), el objetivo de estos estudios en psicología ambiental es reconocer los procesos que median la relación del individuo con el medio ambiente (social y físico). Por ejemplo, fenómenos como el transporte sostenible, el consumo de energía, el manejo de residuos, el uso del agua, entre otros, son desafíos en los que la psicología puede y debe contribuir.

Haciendo referencia al transporte sostenible, la inclusión de factores psicológicos en estudios relacionados a esta temática incrementa la capacidad explicativa de los mismos (Caballero, Franco, Mustaca y Jakovcevic, 2014). Así, teniendo en cuenta los factores psicológicos, junto con variables sociodemográficas y las percepciones frente a la infraestructura disponible, se una comprensión más completa acerca de las conductas de transporte (Hunecke, Blöbaum, Matthies y Höger, 2001; Hunecke, Haustein, Grischkat y Bohler, 2007).

Teniendo en cuenta lo anterior, el objetivo general de este estudio es aportar al creciente conocimiento que busca engranar a las ciencias del comportamiento con las necesidades sociales en materia de transporte sostenible. Esta investigación está enfocada en la identificación de los factores psicológicos asociados al uso habitual de la bicicleta, aportando así a la literatura existente sobre esta materia. Así, el aporte de esta investigación radica en mostrar los factores psicológicos que influencian a los estudiantes universitarios en sus comportamientos de viaje, basándose en investigaciones y modelos que han investigado este tema en otros contextos.

En cuanto a la toma de decisiones con respecto al transporte activo se han utilizado diversos modelos dentro de los cuales se destacan la Teoría de Comportamiento Interpersonal (TIB por sus siglas en inglés, Triandis, 1977,); y la Teoría de Acción Planeada (TAP, Ajzen, 1991), siendo esta última una de las más reconocidas. En el caso de esta investigación se utilizó la TAP, teniendo en cuenta el hábito como factor determinante, puesto que según múltiples estudios, al entender los hábitos de transporte es posible comprender las conductas de transporte (Brette, Buhler, Lazaric y Marechal, 2014; de Brujin, Kremers, Singh, van den Putte, y van Mechelen, 2009; de Brujin y Gardner, 2011; 
Gardner y Abraham, 2008; Muñoz, Monzony Lois, 2013; Muñoz, Monzon y López, 2015).

La TAP, planteada por Ajzen (1985, 1991), postula que la intención de una acción está determinada por tres elementos principales: su actitud hacia el comportamiento, las normas subjetivas que lo rodean y su percepción de control comportamental; y a su vez, la intención de actuar se relaciona con el comportamiento o la ejecución de cierta conducta.

La actitud hacia el comportamiento hace referencia a la evaluación positiva o negativa que puede hacer una persona sobre cierto comportamiento (Sousa, Sanches y Ferreira, 2014). Por ejemplo, preguntas como ¿disfruto montar bicicleta? o afirmaciones como "creo que el uso de la bicicleta como medio de transporte es positivo para nuestra sociedad", pueden revelar actitudes hacia un comportamiento particular hacia el uso de la bicicleta.

Las normas subjetivas, o normas sociales hacen referencia a la percepción del sujeto sobre las presiones externas que existen para incitar a llevar a cabo cierto comportamiento (Ajzen, 1991; Sousa, Sanches y Ferreira, 2014). En este sentido, la norma subjetiva hace referencia a la percepción del sujeto respecto a la aprobación (o reprobación) por parte de los otros frente a determinado comportamiento. Por ejemplo, la percepción de aprobación por parte de la red familiar es un indicador importante de normas subjetivas: ¿apoya tu familia el uso de la bicicleta como medio de transporte? o ¿tiene algún conocido que realice sus viajes en bicicleta? permiten conocer la favorabilidad (o desfavorabilidad) del contexto social del sujeto con respecto al comportamiento.

Por su parte, el control comportamental percibido ( $\mathrm{PBC}$ por sus siglas en inglés) hace referencia a la percepción de control que tiene una persona sobre la probabilidad de llevar a cabo un comportamiento de manera exitosa (Ajzen, 1985; Sousa, Sanches y Ferreira, 2014). Así, preguntas como: "cuento con la capacidad física para realizar viajes diarios en bicicleta" o "conozco las rutas ideales para realizar un recorrido en bicicleta", permiten conocer el grado en que un sujeto se siente en capacidad de llevar a cabo dicho comportamiento.
Así, la TAP permite conocer diferentes factores psicológicos asociados a la comprensión de conductas de transporte. Sin embargo, esta teoría también puede verse complementada por otros componentes que permiten incrementar su poder explicativo. Dependiendo del objetivo de los estudios, es posible encontrar estudios que utilizan únicamente los factores planteados por la TAP (Sousa, Sanches y Ferreira, 2014), así como otros en que se complementa la TAP con factores predictores como los hábitos de transporte (de Brujin y Gardner, 2011; de Brujin et al., 2009; Gardner, 2009; Muñoz, Monzon y Lois, 2013; Muñoz, Monzon y López, 2015), la norma personal (Milković y Stambuk, 2015) y la identidad social (Lois, Moriano y Rondinella, 2015).

Al respecto de esta ampliación de la TAP, Dill y colaboradores (2014) mencionan que la ampliación del modelo es una práctica común dado que la inclusión de factores adicionales ha demostrado ser significativa. Según de Brujin y colaboradores (2009), el hábito es una conducta rutinaria que, como resultado de la repetición, es llevado a cabo de manera casi automática (en vez de ser intencional). En este sentido, el hábito resulta un factor relevante en los estudios de conducta de transporte puesto que puede interferir en el proceso consciente de evaluación y elección de una conducta de viaje, puesto que como establece Gardner (2009) las intenciones tienen una mayor influencia en las acciones cuando no están permeadas por un hábito fuerte (Ouellette y Wood, 1998; Pikora, Giles-Corti, Bull, Jamrozik y Donovan, 2003). Lo anterior significa que al elegir un modo de transporte para realizar un trayecto (una conducta habitual) el hábito puede tener más influencia en la elección que la misma intención, afectando así los modelos de conducta que únicamente tienen en cuenta la intención como elemento predictor de la conducta (Triandis, 1977; Verplanken, Aarts y Knippenberg, 1994).

Finalmente, es importante hacer énfasis en el hecho de que en estos casos la explicación sobre el hábito aumenta cuando se evalúa en conjunto con la intención (intención $\mathrm{x}$ hábito); razón por la cual se justifica la importancia de realizar estudios que midan tanto las variables propuestas por la TAP como el hábito, y no únicamente este último (de Brujin et al., 2007; Gardner, 2009; Heinen, Maat y 
van Wee, 2001). A continuación, se presentan algunos estudios que hacen uso de este tipo de argumentos para realizar sus investigaciones sobre transporte basándose en la TAP y el hábito como factores predictores.

Gardner y Abraham (2008) realizaron una revisión de 23 conjuntos de datos que evaluaban la conducta o intención de uso del automóvil. Los resultados de este estudio confirmaron las asociaciones presupuestas por la TAP entre la intención, las actitudes, las normas subjetivas y el control comportamental percibido $(\mathrm{PBC})$. Además, los autores indicaron que los determinantes especificados por la TAP son objetivos potencialmente útiles para las intervenciones de cambio de comportamiento puesto que, a excepción de la norma subjetiva, estos constructos se correlacionan con el comportamiento.

Por otra parte, Caballero y colaboradores (2014) realizaron una revisión sistemática de la literatura de los últimos 10 años con el propósito de conocer cuáles son las variables psicológicas que mejor predicen el uso de la bicicleta. Dentro de los estudios revisados se encontraron tres (Bamberg, 2014; de Brujin et al., 2009; de Brujin., Kremers y Schaalma, 2005; Gardner, 2009) que utilizan como base modelos que utilizan la TAP e incluyen el hábito como variable predictora. La comparación de estos con otro tipo de modelos permite a los autores concluir que los modelos que incluyen los hábitos cuentan con mayor poder explicativo, alrededor del $50 \%$ de su varianza.

En esta misma línea, de Brujin y colaboradores (2009) realizaron un estudio en el que se incluyeron los hábitos como variable adicional a las propuestas por la TAP, demostrando la relevancia del hábito como correlato más fuerte frente al uso de la bicicleta. De la mano con el resultado anterior, los datos mostraron que cuando el uso de la bicicleta era un hábito fuerte, la intención era un predictor menor del uso de la misma, caso contrario a lo que sucede cuando el uso es un hábito débil.

Por su parte Gardner (2009) mostró que los hábitos tienen una mayor incidencia en los resultados del comportamiento, a pesar de la innegable correspondencia entre hábito e intención; haciendo énfasis en el tipo de viaje que está siendo considerado al realizar estas evaluaciones. Lo anterior puesto que los viajes inestables pueden ser llevados a cabo mediante decisiones más conscientes y deliberativas, mientras que los contextos de viajes estables pueden no necesitar de un nivel de deliberación tan amplio. de Brujin y Gardner (2011) reportan también a la fuerza del hábito como el correlato más fuerte en los ciclistas habituales, y su interacción con la intención; concluyendo que los hábitos más fuertes están relacionados con una asociación más débil entre la intención y el uso de la bicicleta.

En resumen, los anteriores ejemplos permiten ver cómo la inclusión de los hábitos de transporte demuestra ser pertinente en los estudios de conducta de transporte, siendo un complemento de la TAP para evaluar la conducta de transporte de manera integral. A partir de esto, se plantean como objetivos específicos de esta investigación a) describir las características sociodemográficas de estudiantes universitarios frente al uso cotidiano de la bicicleta, b) describir sus hábitos de transporte y c) determinar el grado en que las variables utilizadas en el estudio (provenientes de la Teoría de Acción Planeada) se relacionan con el uso de la bicicleta.

\section{Método}

\section{Participantes}

Se obtuvo acceso a 492 estudiantes universitarios mediante una encuesta virtual, de las cuales 430 encuestas resultaron incluidas en la muestra final. El rango de edad de los participantes estuvo entre 18 y 30 años. El $79.5 \%$ de la muestra estuvo entre los 18 y 22 años con $51.9 \%$ fueron mujeres. La gran mayoría de los encuestados provenía de la ciudad de Bogotá (69.5\%) e indicaron un nivel socioeconómico alto para la mayoría de los encuestados $(77.2 \%$ por encima de la media).

La mayoría de participantes pertenecían a la facultad de Ingeniería (34.9\%), seguidos por la facultad de Ciencias Sociales (13.7\%). En cuanto a los semestres cursados, los participantes mostraron una distribución normal, con una menor participación de estudiantes en los últimos semestres (inicio de estudios: $30 \%$; mitad de carrera: $52 \%$; final de estudios: $18 \%)$. 
En cuanto al contexto académico del hogar, el nivel educativo de los padres y la cantidad aproximada de libros que poseía en el hogar fueron consultados. En general, el nivel educativo alcanzado por la madre fue universitario $(41.9 \%)$ y postgrado $(34.7 \%)$. Para el caso del padre, el nivel universitario fue de ( $35.3 \%$ ) y postgrado de (43\%). Finalmente, en cuanto a la cantidad aproximada de libros en el hogar cerca de la mitad de los encuestados contestó tener más de 100 libros en su hogar (51.6\%).

\section{Procedimiento e Instrumentos}

El comité de ética de la Facultad de Ciencias Sociales de la Universidad de los Andes aprobó la realización del estudio y permitió, una vez consolidado el cuestionario final, el uso de la plataforma Qualtrix, que luego fue distribuido a través de las redes sociales.

\section{Instrumentos}

Para evaluar los factores psicológicos asociados al uso de la bicicleta, se formuló un cuestionario de cinco variables fundamentado en la TAP y variables adicionales (sociodemográficos y hábitos). Un primer conjunto de variables hizo referencia a las características sociodemográficas de los participantes. Datos como la edad, el sexo, la región de origen, la localidad de residencia, el nivel socioeconómico (estrato), los semestres cursados, la facultad, el nivel educativo alcanzado por la madre, el nivel educativo alcanzado por el padre y la cantidad aproximada de libros en el hogar fueron consultados.

El segundo grupo de variables a evaluar en la encuesta estuvo relacionado con los hábitos de transporte. Se realizaron preguntas sobre el tipo de transporte más utilizado y la frecuencia de uso. Así mismo, se indagó acerca de la frecuencia de uso de la bicicleta en particular y el tipo de actividades para las que es utilizada.

Luego, se realizaron preguntas asociadas a los tres factores de la TAP mencionados anteriormente: estos fueron a) actitud hacia el comportamiento, b) norma social y, c) control comportamental percibido. Estas preguntas fueron diseñadas siguiendo la guía de construcción de ítems para la TAP (Ajzen, 2002). Todo el instrumento fue evaluado a través de una escala Likert con las opciones de acuerdo/desacuerdo de siete puntos.

La variable de actitud hacia el comportamiento $(\alpha=0.70)$ fue evaluada a través de afirmaciones con las que los participantes debían manifestar su grado de acuerdo. Frases como: "disfruto montar bicicleta" o "quisiera realizar mis trayectos diarios en bicicleta" fueron utilizadas. Por otra parte, la variable de normas subjetivas $(\alpha=0.74)$ fue evaluada a través de afirmaciones que buscaban dar a conocer el grado de aprobación del uso de la bicicleta como medio de transporte de familiares y grupo de amigos. Así mismo, se buscaba determinar el grado de importancia que tendría para el participante la aprobación de estos dos grupos en caso de decidir utilizar la bicicleta como medio de transporte. En este sentido, frases como "Imagina que decides realizar tus viajes diarios universidad-hogar en bicicleta, indica qué tan de acuerdo estaría tu familia/grupo de amigos" e "Indica el grado de importancia que tendría para ti la aprobación de tu familia/grupo de amigos para realizar tus viajes en bicicleta" fueron planteadas.

Finalmente, el control comportamental percibido $(\alpha=0.75)$ fue evaluado a través de afirmaciones que permitían observar la sensación de control que tenían los participantes sobre ciertas variables externas que influyen en el uso de la bicicleta. Se incluyeron afirmaciones como: "cuento con la capacidad física para realizar viajes diarios en bicicleta" o "conozco lo suficiente la infraestructura de rutas de la ciudad para realizar mis viajes en bicicleta".

El análisis de ítems para la variable Norma Social señaló que no es posible agruparlos dentro de un solo factor, por lo que se decidió evaluar los ítems de manera independiente, siguiendo la sugerencia de Ajzen (2002). Por ende, la variable norma social fue analizada a través de sus cuatro ítems de manera independiente. Para las variables "actitud" y "control comportamental percibido" sí se estableció un factor general. Todos los instrumentos fueron sometidos a procedimientos de evaluación de contenido, mediante juicio de expertos. Una vez diseñado el instrumento, este fue sometido al juicio 
de tres profesionales en psicología con experiencia en metodología y trabajos previos en el área de la psicología ambiental. Estos evaluaron y calificaron la representatividad de cada ítem. Los ítems incluidos en la encuesta final fueron aquellos que arrojaron un índice de concordancia $V$ de Aiken de entre 0.75 y 1.0 entre los jurados. Los ítems que no lograron el índice de concordancia fueron ajustados de acuerdo con los comentarios de los profesionales o eliminados de la encuesta.

También se realizó una prueba piloto con una población similar a la muestra deseada. Posterior a la validación de contenido se realizó una prueba piloto con una muestra de 18 estudiantes, de características similares a la muestra deseada. Se pidió a los participantes responder la encuesta en su totalidad y realizar comentarios sobre claridad de los ítems, diseño y tiempos de respuesta. Como comentarios generales se obtuvieron sugerencias frente a la claridad en la definición la variable sexo, un par de ítems de respuesta invertida, y el orden de presentación de los ítems.

\section{Análisis de datos}

La unidad de análisis establecida fue entonces el uso de la bicicleta, utilizado como la variable dependiente durante todo el análisis. Posteriormente, se calcularon los estadísticos descriptivos y las correlaciones. Más adelante se realizaron pruebas $t$ de Student luego de determinar la normalidad de las variables mediante la prueba de KolmogorovSmirnov. Finalmente, se realizó una taxonomía de modelos derivada de análisis de regresión múltiple para determinar el grado en que las variables descritas se relacionan con el uso de la bicicleta. Se estableció un valor de significancia conservador de 0.05 y un tamaño del efecto pequeño $(<0.5)$.

\section{Resultados}

Como muestra la Tabla 1, la actitud hacia la bicicleta presentó una media de 5.64 ( $D E=1.03)$. La variable de Control Comportamental Percibido tiene una media de 4.20 ( $D E=1.17)$. La Norma Social fue evaluada a través de los cuatro ítems que hacen referencia a dos grupos particulares: amigos y familia. El acuerdo de la familia frente al uso de la bicicleta tiene una media de 4.19 ( $D E=2.25$ ), la importancia de la aprobación de la familia tiene una media de $3.50(D E=2.00)$. En cuanto al grupo de amigos, el acuerdo frente al uso de la bicicleta tiene una media de 5.60 ( $D E=1.87$ ), y la aprobación de este grupo tiene una media de 1.94 ( $D E=1.35)$.

En cuanto a los hábitos de transporte, los participantes indicaron que el transporte público (46\%) y el carro $(36 \%)$ son los medios más utilizados para realizar los trayectos universidad-hogar. Por su parte el $12 \%$ de los mismos contestó realizar sus viajes en bicicleta. En cuanto a la frecuencia de uso del anterior medio, el $44 \%$ reportó utilizarlo todos los días, y el $38 \%$ de 4 a 6 días a la semana. Finalmente se consultó sobre el tipo de actividades para las que los participantes utilizaban la bicicleta. Como posibles actividades de uso se establecieron los a) viajes cortos (33\%); b) la ciclovía (47\%); c) otros (11\%) y d)

Tabla 1. Estadísticos descriptivos variables modelo

\begin{tabular}{lccccc}
\hline & Media & DE & Curtosis & Máximo & Mínimo \\
\hline Actitud & 5.64 & 1.03 & -0.79 & 5.40 & 1.60 \\
PBC & 4.20 & 1.17 & 0.02 & 6.00 & 1.00 \\
AcuFami & 4.19 & 2.25 & 0.52 & 12.00 & 1.00 \\
AproFami & 3.50 & 2.01 & 0.58 & 12.00 & 1.00 \\
AcuAmi & 5.60 & 1.87 & 4.21 & 27.00 & 1.00 \\
AproAmi & 1.94 & 1.35 & 1.50 & 6.00 & 1.00 \\
\hline
\end{tabular}

*AcuFami: Acuerdo Familia; AproFami: Aprobación Familia; AcuAmi: Acuerdo Amigos; AproAmi: Aprobación Amigos.

Fuente: elaboración propia. 
nunca la uso ( $28 \%$ ), donde los participantes podían elegir múltiples actividades.

Dentro del análisis correlacional realizado entre el uso de la bicicleta y las variables sociodemográficas, las variables de edad $(r=0.304, p<0.001)$ y semestres cursados $(r=0.239, p<0.001)$ mostraron ser significativas.

Por otra parte, dentro del análisis correlacional realizado entre el uso de la bicicleta y las variables del modelo (Tabla 2) se obtuvo que el uso de la bicicleta correlacionó positivamente con actitud $(r=$ $0.609, p<0.001$ ), control comportamental percibido $(r=0.657, p<0.001)$, y norma social. Esta última indica correlaciones en cuanto al acuerdo del grupo frente al uso de la bicicleta, tanto entre el grupo de amigos $(r=0.164, p<0.001)$ como la familia $(r=0.349, p<0.001)$. Vale resaltar que, dentro de la norma social, la importancia de la aprobación de la familia $(r=-0.116, p<0.016)$ muestra una correlación inversa con el uso de la bicicleta.

Para evaluar las variables categóricas: sexo y facultad, se realizaron tablas cruzadas que permitieron observar el uso de la bicicleta en las diferentes categorías. En cuanto a la facultad, 70 participantes pertenecientes a la facultad de Ingeniería indicaron usar la bicicleta todos los días, seguidos por la facultad de Ciencias Sociales con 31 personas. Por otra parte, 141 mujeres frente a 73 hombres reportaron usar la bicicleta todos los días (Tabla 3). Teniendo en cuenta la diferencia de la cifra anterior fue realizada una prueba $t$ para comparar los dos grupos, indicando que sí existen diferencias significativas entre sexo y uso de la bicicleta y que el uso es mayor en las mujeres $\left(t_{(428)}=-6.514 ; p<0.001 ; d=0.71\right)$.

Con el objetivo de determinar la asociación entre las variables, se realizaron regresiones para determinar la incidencia de las variables en la capacidad explicativa del modelo. Como muestra la Tabla 4, el primer modelo, que incluyó la edad y el sexo explicó el $16 \%$ de la varianza del uso de la bicicleta. El siguiente modelo evaluó la variable control comportamental percibido, que explicando un $43 \%$ de la varianza. En el modelo siguiente, en el cual se sumó la actitud al control comportamental percibido se explicaba el $47 \%$ de la varianza. Así, el último modelo, que incluía todas las variables explicaba el $49.6 \%$ de la varianza del uso de la bicicleta.

Tabla 2. Correlaciones variables del modelo

\begin{tabular}{lcccccc}
\hline & Actitud & PBC & AcuFami & AproFami & AcuAmi & AproAmi \\
\hline Uso Bicicleta & $0.609^{* *}$ & $0.657^{* *}$ & $0.349^{* *}$ & $-0.116^{*}$ & $0.164^{* *}$ & -0.013 \\
Actitud & & $0.699^{* *}$ & $0.399^{* *}$ & -0.034 & $0.288^{* *}$ & 0.071 \\
PBC & & & $0.461^{* *}$ & $-0.117^{*}$ & $0.288^{* *}$ & 0.000 \\
AcuFami & & & & $-0.312^{* *}$ & $0.282^{* *}$ & 0.009 \\
AproFami & & & & & 0.029 & $0.358^{* *}$ \\
AcuAmi & & & & & & 0.037 \\
\hline
\end{tabular}

*AcuFami: Acuerdo Familia; AproFami: Aprobación Familia; AcuAmi: Acuerdo Amigos; AproAmi: Aprobación Amigos

Fuente: elaboración propia.

Tabla 3. Frecuencia de uso vs. Sexo

\begin{tabular}{ccccccccc}
\hline & & Todos los días & $\begin{array}{c}\text { 4-6 veces por } \\
\text { semana }\end{array}$ & $\begin{array}{c}\text { 2-3 veces por } \\
\text { semana }\end{array}$ & $\begin{array}{c}\text { Una vez por } \\
\text { semana }\end{array}$ & Nunca & Total \\
\hline Sexo: & $\mathrm{F}$ & 141 & 45 & 22 & 9 & 6 & 223 \\
& $\mathrm{M}$ & 73 & 43 & 53 & 24 & 14 & 207 \\
Total & 214 & 88 & 75 & 33 & 20 & 430 \\
\hline
\end{tabular}

Fuente: elaboración propia. 
Tabla 4.

Taxonomía de modelos GLM

\begin{tabular}{|c|c|c|c|c|c|c|c|c|}
\hline \multirow[b]{2}{*}{ M } & \multicolumn{2}{|c|}{ M1 } & \multicolumn{2}{|c|}{ M2 } & \multicolumn{2}{|c|}{ M3 } & \multicolumn{2}{|c|}{ M4 } \\
\hline & B & SE & B & SE & B & SE & $B$ & SE \\
\hline Intercepto & -0.03 & 0.22 & $-0.81^{*}$ & 0.16 & $-1.83^{*}$ & 0.22 & $-2.24^{*}$ & 0.24 \\
\hline Edad & $0.14^{*}$ & 0.02 & & & & & $0.08^{*}$ & 0.01 \\
\hline Sexo & $0.66^{*}$ & 0.10 & & & & & & \\
\hline pcb & & & $0.661^{*}$ & 0.03 & $0.45^{*}$ & 0.04 & $0.41^{*}$ & 0.04 \\
\hline Actitud & & & & & $0.33^{*}$ & 0.05 & $0.33^{*}$ & 0.05 \\
\hline$R^{2}$ Ajust. & \multicolumn{2}{|c|}{0.167} & \multicolumn{2}{|c|}{0.43} & \multicolumn{2}{|c|}{0.47} & \multicolumn{2}{|c|}{0.49} \\
\hline
\end{tabular}

*Significativo a un nivel de 0.05 .

Fuente: elaboración propia.

\section{Discusión}

Este estudio buscó explorar los factores psicológicos asociados al uso de la bicicleta a la luz de la teoría de acción planeada, teniendo en cuenta los hábitos de transporte. Para determinar el grado en que las variables se relacionan con el uso de la bicicleta se realizaron análisis de regresión múltiple. Los resultados del análisis muestran que el modelo 4 explica el $49.6 \%$ de la varianza explicada del modelo.

Esto significa que las personas de mayor edad, mayor actitud y mayor control comportamental percibido son la mejor caracterización significativa que se puede tener de los estudiantes biciusuarios. Lo anterior está en línea con los resultados encontrados en la literatura revisada (de Brujin et al., 2009; Gardner, 2009; Gardner y Abraham, 2008) pues dos de los elementos de la TAP aparecen como significativos en este modelo. En este sentido, esta investigación aporta evidencia a la utilidad de la TAP para estudios de conductas de transporte relacionados con el uso de modos sostenibles.

Sin embargo, es importante resaltar que dentro del modelo, el control comportamental percibido es el correlato más fuerte con el uso de la bicicleta, pues da cuenta de un $43 \%$ de la varianza explicada del modelo. Esto implicaría que la percepción de las personas frente a elementos como la capacidad física, la infraestructura de la ciudad y las condiciones climáticas resultan determinantes frente al uso de la bicicleta, por lo que podría sugerirse que las futuras campañas dirigidas a la promoción del uso de la bicicleta en los estudiantes universitarios estén orientadas hacia elementos relacionados al control comportamental percibido.

Al realizarse la taxonomía de modelos incluyendo los ítems de norma social estos no resultaron ser estadísticamente significativos. Sin embargo, dentro del análisis correlacional se identificaron asociaciones significativas para los ítems relacionados al acuerdo del grupo de amigos y familia. Esto significa que los participantes perciben que su grupo de amigos y familia estaría de acuerdo con el uso de la bicicleta como medio de transporte. Sin embargo, en cuanto a la asociación entre la aprobación del grupo y el uso, esta parece no influir en la toma de decisiones de los participantes pues incluso para el caso de la aprobación familiar la correlación se mostró negativa.

Respecto a los hábitos de transporte, los datos recolectados indican que la frecuencia de uso de los medios más utilizados está compuesta en un $82 \%$ por participantes que los usan mínimo cuatro veces a la semana. En otras palabras, en el $82 \%$ de los casos, el medio que el participante seleccionó como el más utilizado (por ejemplo, auto particular) es utilizado mínimo cuatro veces a la semana, mostrando una mayor fortaleza del hábito al momento de elegir el modo de transporte. En consecuencia con lo señalado (Brette et al., 2014; Gardner, 2008), 
los datos permiten inferir que las decisiones deliberadas de modo de transporte se dan pocas veces y estas se traducen en hábitos poco alterables, pues una vez elegido el medio lo más probable es que se haga uso del mismo de manera cotidiana.

Esto implica un reto en la promoción de modos de transporte sostenible, pues los hábitos de transporte pueden llegar a tener una incidencia mayor a la intención a la hora de elegir un modo de transporte. En este sentido, la literatura sobre el fomento de medios de transporte sostenibles puede orientar en gran medida las estrategias para desincentivar el uso de medios como el vehículo particular. Ejemplo de lo anterior es el concepto denominado ventana de oportunidad mencionado por Brette y colaboradores (2014), que hace referencia a la existencia de momentos particulares en la vida de un individuo en los que es más probable que se dé un cambio de hábitos. El caso del programa Portland's SmartTrips Welcome Program (2013) es una muestra de la implementación de este concepto. Este programa busca fomentar comportamientos positivos en transporte en los ciudadanos, enfocándose en los nuevos residentes de la ciudad, aprovechando los cambios de hábitos de movilización; siendo más efectivo que hacerlo en personas quienes siguen con su estilo de vida cotidiano (antiguos residentes).

Como plantean Gardner y Abraham (2008) los determinantes especificados por la TAP son objetivos potencialmente útiles para las intervenciones de cambio de comportamiento puesto que estos constructos (excepto la norma subjetiva) se correlacionan con el comportamiento. En este sentido, la información recolectada permite dar un primer paso hacia el planteamiento de intervenciones para el fomento del uso de la bicicleta en estudiantes universitarios, sustentadas en elementos de la psicología.

Como primer elemento a utilizar está la caracterización de los estudiantes y sus hábitos de transporte, lo que permite identificar elementos detallados sobre la población a la que pueden/deben dirigirse los esfuerzos. Ejemplo de lo anterior es identificar las facultades con mayor tendencia a hacer uso de la bicicleta como medio de transporte y así evaluar si la mejor estrategia de divulgación de campañas sería una campaña unificada para todos los estudiantes, o campañas particulares para cada facultad. Por otro lado, y teniendo en cuenta el caso del Portland's SmartTrips Welcome Program (2013), es posible sugerir que la población objetivo de las campañas de promoción sea la de estudiantes recién ingresados que utilicen, o planeen utilizar el carro como medio de transporte. De esta manera, la campaña cumpliría la función de mitigar en cierta medida la generación de hábitos de transporte que pueden ser más difíciles de modificar una vez los estudiantes se adapten a sus nuevas rutinas de viaje.

En segundo lugar, conocer los hábitos de transporte de los estudiantes permiten identificar las actividades para las que los estudiantes utilizan la bicicleta. Esto permitiría inferir entonces que la principal barrera para el uso de la bicicleta no parecería ser el desconocimiento (nunca usar/nunca haber usado) de la bicicleta, sino por el contrario la asociación de la misma a otro tipo de actividades por fuera del transporte cotidiano. En este sentido sería posible recomendar que las campañas de promoción no estén enfocadas a impulsar el acercamiento a la bicicleta como tal, sino al cambio de uso de la misma para otro tipo de actividades. Por ejemplo, promover los grupos estudiantiles relacionados al transporte a la universidad en bicicleta podría ser una estrategia favorable. Siendo consecuentes con la información obtenida, de generarse los grupos, estos deberán ser orientados hacia las barreras que son percibidas por los estudiantes (en su mayoría asociadas al control comportamental percibido). Así, los grupos deberían estar en capacidad de apoyar a los estudiantes frente a cuestiones como el reconocimiento de las rutas de la ciudad, estrategias para poder afrontar las condiciones climáticas de la ciudad y la generación de grupos de apoyo para fortalecer la sensación de seguridad, entre otros temas.

Como fue mencionado anteriormente, el modelo propuesto para la presente investigación explicó el $49.6 \%$ de la varianza. De lo anterior se desprenden dos recomendaciones principales: 1) ampliar la capacidad explicativa del modelo, lo que implica preguntarse por otras variables faltantes; y 2) utilizar diferentes modelos para esta misma investigación, que permitan nutrir el conocimiento frente a las conductas de transporte sostenible. 
Por último, para futuras investigaciones sería importante complementar las evaluaciones cuantitativas con componentes cualitativos, por ejemplo, entrevistas a profundidad que permitan conocer las razones de fondo frente a elementos que pueden parecer imparciales en preguntas de selección múltiple. Con detalles como los que generarían evaluaciones cuantitativas y cualitativas podrían realizarse estudios más específicos en cuanto a las poblaciones objetivo. Por ejemplo, podrían enfocarse futuros estudios a las poblaciones que no utilizan la bicicleta para realizar sus trayectos, a aquellos que utilizan el carro particular, o aquellos estudiantes pertenecientes a las facultades que puntuaron más bajo en el ítem de uso de la bicicleta.

A manera de conclusión, los retos actuales de sostenibilidad en nuestra sociedad se relacionan con nuestro comportamiento. Así, generar estrategias eficaces para hacer frente a estos retos implica tener una mayor comprensión sobre los factores que influyen en los procesos de toma decisiones y las acciones que los siguen. Como fue expuesto a lo largo de esta investigación, en el caso de los estudios de transporte sostenible, la inclusión de factores psicológicos permite desarrollar estrategias comprensivas para orientar los esfuerzos (es decir, políticas públicas, leyes, campañas, entre otros) para promover conductas orientadas a una mayor sustentabilidad.

\section{Referencias}

Ajzen, I. (1985). From intentions to actions: A theory of planned behavior. En J. Kuhl y J. Beckman (Eds.). Action-control: From cognition to behavior, 11-39. Heidelberg: Springer.

Ajzen, I. (1991). The theory of planned behavior. Organizational Behavior and Human Decision Processes, 50(2), 179-211. http://doi. org/10.1016/0749-5978(91)90020-T

Ajzen, I. (2002). Constructing a TpB Questionnaire: Conceptual and Methodological Considerations. Recuperado de https://pdfs.semanticscholar.org/0574/b20bd58130dd5a961f1a2db10fd1fcbae95d.pdf
Baird, B. (2015). Informing Public Policy with Social and Behavioral Science. Issues in Science y Technology, 31(3), 31-34.

Bamberg, S. (2014) Psychological Contributions to the Development of Car Use Reduction Interventions. En: Gärling, T., Ettema, D., Friman, M. (eds) Handbook of Sustainable Travel. Dordrecht: Springer.

Brette, O., Buhler, T., Lazaric, N. y Marechal, K. (2014). Reconsidering the nature and effects of habits in urban transportation behaviour (Working Paper No. 2014-10). Groupe de Recherche en Droit, Economie, Gestion. Recuperado de http://www.gredeg.cnrs.fr/ working-papers/GREDEG-WP-2014-10.pdf

Caballero, R., Franco, P., Mustaca, A. y Jakovcevic, A. (2014). Uso de la Bicicleta como Medio de Transporte: Influencia de los Factores Psicológicos. Una Revisión de la Literatura. Psicología Ambiental, 45(3), 316-324.

De Brujin, G. y Gardner, B. (2011). Active commuting and habit strength: An interactive and discriminant analyses approach. American Journal of Health Promotion, 25(3), 27-34. http;//doi. org/10.4278/ajhp.090521-QUAN-170

De Brujin, G., Kremers, S., Singh, A., van den Putte, B., van Mechelen, W. (2009). Adult active transportation: adding habit strength to the Theory of Planned Behaviour: the case of travel habit. American Journal of Preventive Medicine, 36(3), 189-194. http://doi.org/10.1016/j. amepre.2008.10.019

De Brujin, G., Kremers, S., de Vet, E., Nooijer, D., van Mechelen, W. y Brug, J. (2007). Does habit strength moderate the intention-behaviour relationship in the theory of planned behaviour? the case of fruit consumption. Psychological Health, 22, 899-919. http://doi. org/10.1080/14768320601176113

De Brujin, G., Kremers, S. y Schaalma, H., van Mechelen, W. y Brug, J. (2005). Determinants of adolescent bicycle use for transportation and 
snacking behavior. Preventive Medicine, 40(6), 658-667. http://10.1016/j.\&pmed.2004.09.003

Dill, J., Mohr, C. y Ma, L. (2014). How Can Psychological Theory Help Cities Increase Walking and Bicycling? Journal of the American Planning Association, 80(1), 36-51. http://doi. org/10.1080/01944363.2014.934651

Gardner, B. (2009). Modelling motivation and habit in stable travel mode contexts. Transportation Research Part F, 12(1), 68-76. http://doi. org/10.1016/j.trf.2008.08.001

Gardner, B. y Abraham, C. (2008). Psychological correlates of car use: A meta-analysis. Transportation Research Part F: Traffic Psychology and Behaviour, 11(4), 300-311. http://doi.org/10.1016/j.trf.2008.01.004

Gifford, R. (2007). Environmental Psychology and Sustainable Development: Expansion, Maturation, and Challenges. Journal of Social Issues, 63(1), 199-212. http://doi. org/10.1111/j.1540-4560.2007.00503.x

Heinen, E., Maat, K. y van Wee, B. (2001). The role of attitudes toward characteristics of bicycle commuting on the choice to cycle to work over various distances. Transportation Research, Part D: Transport and Environment, 16(2), 102109. http://doi.org/10.1016/j.trd.2010.08.010

Hunecke, M., Blöbaum, A., Matthies, E. y Höger, R. (2001). Responsibility and environment-ecological norm orientation and external factors in the domain of travel mode choice behavior. Environment \& Behavior, 33(6), 830-852. http://doi.org/10.1177/00139160121973269

Hunecke, M., Haustein, S., Grischkat, S. y Bohler, S. (2007). Psychological, sociodemographic and infrastructural factors as determinant of ecological impact caused by mobility behavior. Journal of Environmental Psychology, 27(2), 277-292. http://doi.org/10.1016/j. jenvp.2007.08.001

Lois, D., Moriano, J. y Rondinella, G. (2015). Cycle commuting intention: A model based on theory of planned behaviour and social identity. Transportation Research Part F: Traffic Psychology and Behaviour, 32, 101-113. http:// doi.org/10.1016/j.trf.2015.05.003

Milković, M. y Stambuk, M. (2015). To Bike or not to Bike? Application of the Theory of Planned Behavior in Predicting Bicycle Commuting Among Students in Zagreb. Psihologijske teme, 24(2), 187-205. https://hrcak.srce.hr/142128

Moser, G. (1998). Psicología Ambiental. Estudos de Psicologia, 3(1), 121-130.

Moser, G. (2003). La Psicología Ambiental en el Siglo 21: El Desafío del Desarrollo Sustentable. Revista de Psicología de la Universidad de Chile, 7(2), 11-17.

Muñoz, B., Monzon, A.y Lois, D., (2013). Cycling habits and other psychological variables affecting commuting by bicycle in Madrid, Spain. Transportation Research Record: Journal of Transportation Research Board, 2382(1), 1-9. http://doi.org/10.3141/2382-01

Muñoz, B., Monzon, A. y López, E. (2015). Transition to a cyclable city: Latent variables affecting bicycle commuting. Transportation Research Part A: Policy and Practice, 84, 4-17. http:// doi.org/10.1016/j.tra.2015.10.006

Ouellette, J. y Wood, W. (1998). Habit and intention in everyday life: the multiple processes by which past behavior predicts future behavior. Psychological Bulletin, 124(1), 54-74. http://doi. org/10.1037//0033-2909.124.1.54

Pikora, T., Giles-Corti, B., Bull. F., Jamrozik K. y Donovan, R. (2003). Developing a framework for assessment on the environmental determinants of walking and cycling. Social Science \& Medicine, 56(8), 693-703. http://doi. org/10.1016/S0277-9536(02)00163-6

Portland's SmartTrips Welcome Program. (2013). Recuperado el 8 de mayo de 2017 de http://www.toolsofchange.com/en/ case-studies/detail/658 
Sousa, A., Sanches, S. y Ferreira, M. (2014). Perception of barriers for the use of bicycles. Social and Behavioral Sciences, 160, 304 - 313. http://doi.org/10.1016/j.sbspro.2014.12.142

Triandis, H. (1977). Interpersonal behavior. Monterey, ca: Brooks-Cole.
Verplanken, B., Aarts, H. y Knippenberg, A. (1994). Habit, information acquisition, and the process of making travel mode choices. European Journal of Social Psychology, 27(5), 539-560. http://doi.org/10.1002/ (SICI)1099-0992(199709/10)27:5<539::AIDEJSP831>3.0.CO;2-A 